\title{
Training General Practitioners in the Treatment of Functional Somatic Symptoms: Effects on Patient Health in a Cluster-Randomised Controlled Trial (the Functional Illness in Primary Care Study)
}

\author{
Tomas Toft $^{\mathrm{a}}$ Marianne Rosendal $^{\mathrm{b}} \quad$ Eva $\left.\varnothing_{\mathrm{rn}} \varnothing\right|^{\mathrm{a}}$ Frede Olesen $^{\mathrm{b}}$ \\ Lisbeth Frostholm ${ }^{\mathrm{a}}$ Per Fink ${ }^{\mathrm{a}}$ \\ ${ }^{a}$ Research Clinic for Functional Disorders and Psychosomatics, Aarhus University Hospital, and \\ ${ }^{b}$ Research Unit for General Practice, Aarhus University, Aarhus, Denmark
}

\section{Key Words}

Somatoform disorders $\cdot$ Mental disorders $\cdot$ Primary health care $\cdot$ Family practice $\cdot$ Randomised controlled trials . Disability evaluation - Treatment outcome - Medically unexplained symptoms • Questionnaires

\begin{abstract}
Background: Patients with medically unexplained or functional somatic symptoms (FSS) are prevalent in primary care. In this pragmatic cluster-randomised controlled trial we aimed to test the effect of a training programme (The Extended Reattribution and Management model) for general practitioners (GPs) in the treatment of FSS. Methods: 38 participating GPs were randomised to the control group or the training group. The GPs included consecutive 18- to 65-yearold patients presenting during a 3-week period for new health complaints. We assessed a stratified subsample with the psychiatric interview Schedules of Clinical Assessment in Neuropsychiatry. Of 701 patients interviewed, 350 fulfilled the diagnostic criteria for any ICD-10 somatoform disorder (SD) and 111 presented FSS without fulfilling these criteria
\end{abstract}

(sub-threshold SD). Patients completed questionnaires at baseline and after 3, 12 and 24 months. The questionnaires included assessment of health status (36-item Medical Outcomes Study Short Form; SF-36), health anxiety (Whiteley-7) and physical symptoms (Symptom Check List-90, somatization subscale). Results: Patients with SD consulting trained GPs improved more on our primary outcome of physical functioning than patients consulting control GPs at the 3month follow-up ( $p=0.004)$, but the improvement was not statistically significant at later follow-up. We found no significant differences in improvement between patients with SD and those with sub-threshold SD. Results for other SF-36 subscales, physical symptoms and health anxiety only showed statistically significant differences between the intervention and control groups for patients with SD; patients consulting trained GPs had less improvement in vitality, health anxiety and physical symptoms at 24 months compared with the control group. Conclusions: GP training may accelerate improvement in physical functioning for patients with SD. However, the effect is small and may not be clinically significant.

Copyright $\odot 2010$ S. Karger AG, Basel

\section{KARGER}

Fax +41613061234 E-Mail karger@karger.ch www.karger.com
(C) 2010 S. Karger AG, Base

$0033-3190 / 10 / 0794-0227 \$ 26.00 / 0$

Accessible online at:

www.karger.com/pps
Per Fink

Research Clinic for Functional Disorders and Psychosomatics

Aarhus University Hospital, Noerrebrogade 44

DK-8000 Aarhus C (Denmark)

Tel. +45 8949 4310, Fax +45 8949 4340, E-Mail aarhus.ffl@ rm.dk 


\section{Introduction}

Primary care patients frequently present with physical symptoms which cannot be explained by any known medical condition, i.e. medically unexplained or functional somatic symptoms (FSS) [1-7]. The severity of FSS ranges from mild transient cases to severe and chronic somatoform disorders (SDs) [8]. SDs can be disabling for patients [5], they are costly for society due to sick leave, loss of working years and high health care utilisation $[5$, 9], and patients risk being exposed to iatrogenic harm in bio-medically focused health care systems $[5,9,10]$. Many general practitioners (GPs) find encounters with FSS patients difficult [11] and express a need for improved treatment strategies [12].

Patients with FSS may be treated effectively with psychotherapy provided by specialists [13-16]. However, for capacity reasons and because early intervention could be more cost-effective and prevent chronification of the condition, pragmatic primary care trials with GPs as therapists are needed [17]. So far, only a few such studies have been published, of which most include reattribution techniques [18-24]. The study designs and patients included have been very heterogeneous, and the results have been conflicting.

This study describes the effects of a treatment model developed for GPs and based on both the original reattribution model and principles from cognitive therapy, called The Extended Reattribution and Management (TERM) model [25]. The model has previously demonstrated positive effects on doctors' attitudes toward patients with FSS $[26,27]$ and possibly also on patients' satisfaction with care $[22,28]$, but effects on patient health and health care utilisation remain uncertain.

The objective of this pragmatic randomised controlled trial (RCT) was to evaluate the effects of GP training on patients with FSS classified as SD and sub-threshold SD with regard to the patients' physical and mental health.

\section{Methods}

The study was carried out between 2000 and 2002 in Aarhus County in Denmark as a cluster-RCT with a 2 -year follow-up. Randomisation and intervention were performed at the medical practice level and outcome was measured at the patient level. Aarhus County is a mixed rural and metropolitan area with a population of 600,000 served by 431 GPs in 271 practices. In Denmark, $98 \%$ of the population is listed with a general practice, and these act as gatekeepers for the specialised health care system.
Inclusion and Randomisation of GPs

All GPs in Aarhus County were invited to participate, and 40 GPs (9.3\%) from 30 practices accepted. Practices were stratified according to the number of GPs in the practice and block-randomised to the control or intervention group. A neutral party performed the randomisation by drawing lots with practice numbers from an opaque bag. Two GPs from the intervention group were excluded from the study; one reported sick at the residential course and one failed to include any patients. 38 GPs from 28 practices completed the study (fig. 1).

\section{Intervention}

The GPs in the intervention group were trained in the TERM model (table 1) [25]. The training programme included a 2-day residential course followed by 3 weekly 2 -hour evening sessions with analysis of the GPs' videotaped consultations with their own patients, a booster meeting after 3 months and an outreach visit by a supervisor after 6 months. The model provides GPs with both theoretical knowledge about FSS and the practical skills necessary for the assessment and treatment of FSS patients. Table 1 illustrates the 5 stages in the treatment of FSS patients and the applied interviewing techniques. The manual and training material are available on request. The GPs in the control group were only informed about the definitions of FSS and SD and the contents of the questionnaires.

\section{Inclusion of Patients}

Consecutive patients aged 18-65 years consulting their GP for a new health problem were included during a 3-week period. $\mathrm{Pa}$ tients were excluded if they were of non-Scandinavian origin or too ill or demented to answer the questionnaires. Patients consented to participate after receiving written and oral information on the study. Patients were not informed about the assignment of their GP to either the intervention or control group. The GPs were reimbursed for included patients.

\section{Study Phase 1: Baseline Data}

Before the consultation with their GPs, the patients filled in questionnaires, including the 36-item Medical Outcomes Study Short Form (SF-36) [29], assessing health status and disability, a screening questionnaire consisting of the 8-item Symptom Check List (SCL-8) [30] for mental disorders, the 12-item SCL Somatization Subscale (SCL-SOM) [31] for somatic symptoms, the 7-item Whiteley index (Whiteley-7) [32] for health anxiety and the CAGE questions about alcohol addiction [33]. The GPs reported on symptom chronicity at the baseline consultation. Information about the GPs' sociodemographics and education was obtained in a parallel study on GPs' postgraduate education in Aarhus County [34].

\section{Study Phase 2: Diagnostic Interviews}

A random sample of every ninth consecutive patient and all high-scorers on the screening questionnaires was selected for the psychiatric diagnostic interview Schedules of Clinical Assessment in Neuropsychiatry (SCAN) [35]. Patients with a positive score on 2 or more items on the dichotomised scales of Whiteley7, SCL- 8 or CAGE, or on 4 or more items on the dichotomised SCL-SOM were considered high-scorers. A total of 894 patients were selected for the interview, comprising 686 high-scorers, 119 randomly selected patients and 89 patients who were both high-
Toft/Rosendal/Ørnbøl/Olesen/ Frostholm/Fink 
Table 1. The TERM model [25]

\section{Treatment steps}

I Understanding

1 Take a full symptom history

2 Explore emotional cues

3 Inquire directly about symptoms of anxiety and depression

4 Explore life events and stressors

5 Explore functional level

6 Explore the patient's health beliefs

7 Explore the patient's expectations

8 Make a brief, focused physical examination

II The physician's expertise and acknowledgement

9 Provide feedback on the results of the assessment

10 Acknowledge the reality of the symptoms

11 Make clear that there is no (or that there is indeed) indication for further examination

III Negotiating a new model of understanding

12 Simple explanations

13 Practical demonstrations

14 Dealing with severe cases

IV Negotiating further treatment

15 Sum up agreements

16 Agree on specific objectives, contents and form of future course

17 Acute cases: no further appointments

18 Sub-acute cases: therapy, regular scheduled appointments

$\mathrm{V}$ Management of chronic cases

a Status consultation

b Observe general principles about management of patients with chronic disorders focussing on physical, psychological, pharmacological and administrative aspects

\author{
Interviewing techniques \\ Socratic questioning \\ Neutral and sincerely curious \\ Open questions \\ Encouragement (facilitation) \\ Keep the focus \\ Frequent summaries \\ Empathy/emotional feedback \\ Roll with resistance \\ Empowerment
}

scorers and randomly selected. Of these 894 patients, 701 (78.4\%) agreed to be interviewed (fig. 1). Six doctors, all with more than 2 years of psychiatric residency and formally trained and certified, conducted the SCAN interviews. Inter-rater correlation was tested on 8 patients, resulting in a kappa value of 0.82 for ICD-10 SD. The interviewers were blinded to the patients' randomisation and responses to questionnaires.

\section{Study Phase 3: Follow-Up}

After 3, 12 and 24 months, all participating patients were sent a questionnaire that included the same items about health as the baseline questionnaire. Participants received a reminder if they failed to answer within 3 weeks.

Treatment of Functional Somatic

Symptoms: Effects on Patient Health
Outcome Measures

The primary outcome measure was the change in physical functioning as measured by the SF-36 subscale physical functioning. Secondary outcome measures were changes in mental and social functioning, perceived health, vitality and pain as measured by the corresponding SF-36 subscales, changes in somatic symptoms as measured by the SCL-SOM and changes in health anxiety as measured by the Whiteley-7.

\section{Analyses}

Three groups of patients with FSS were analysed: (1) patients fulfilling the diagnostic criteria for an ICD-10 SD (F44.4-F48.0; $\mathrm{n}=350$ ), (2) the subgroup of SD patients with somatisation disorder $(\mathrm{F} 45.0 ; \mathrm{n}=117)$ and (3) patients complaining of 2 or more incapacitating FSS but not fulfilling the diagnostic criteria for an ICD-10 SD (n = 111; sub-threshold SD).

\section{Statistical Methods}

Power calculations were performed using a 2 -sided $t$ test (type 1 error 0.05 , power 0.8 ) and a test-retest correlation of 0.60 between repeated measurements at the patient level. In order to identify a treatment effect of 10 points' difference for the SF-36 physical functioning subscale, inclusion of 55 patients was necessary. We allowed for a $25 \%$ prevalence of SDs, a doctor detection rate of $50 \%$, a patient follow-up rate of $70 \%$ and a surplus of $20 \%$ because of the cluster effect. This resulted in the need to include at least 755 patients in each arm of the study at baseline.

Data on GPs were compared for participating doctors and non-participants using the likelihood ratio (LR) test in a multiple logistic regression model adjusting for gender, age, type of clinic, seniority as a GP, postgraduate psychiatric training and participation in communication courses.

At the patient level, sociodemographics were compared in the intervention and control groups using a t test or the WilcoxonMann-Whitney rank sum test for continuous variables and the $\chi^{2}$ test for categorical data. Multiple logistic regression methods were used to analyse the sociodemographic characteristics of patients with missing data during follow-up.

Multiple linear regression methods for outcome at the 3 follow-up points were used to estimate the effect of the intervention, calculated as the difference in outcome between the intervention and control groups, adjusting for age, gender, chronic disease and condition at baseline. These analyses were corrected for the cluster effect at the GP level.

We employed 3 different methods for handling missing data in the analyses: (1) patients who responded to all follow-up questionnaires (respondents), (2) respondents plus patients providing at least baseline information on condition and GP information about chronicity (missing data for outcome was set to zero), and (3) respondents plus patients who responded to some of the questionnaires and on sociodemographic data (multiple imputed data).

We applied the multiple imputation technique called the predictive method, with the number of imputed datasets equal to 5 for the component scores and the physical functioning sum score of the SF-36 and equal to 10 for the remaining sum scores [36]. As covariates in the regression model of the predictive method, complete variables were used such as age, gender, whether the screening questionnaire was revealed to the GP, high score status based 
Fig. 1. Inclusion and follow-up for GPs and patients. ${ }^{1}$ One GP fell ill and could not attend the residential course and 1 GP failed to include any patients. ${ }^{2}$ Percentage of patients replying compared to number at baseline.

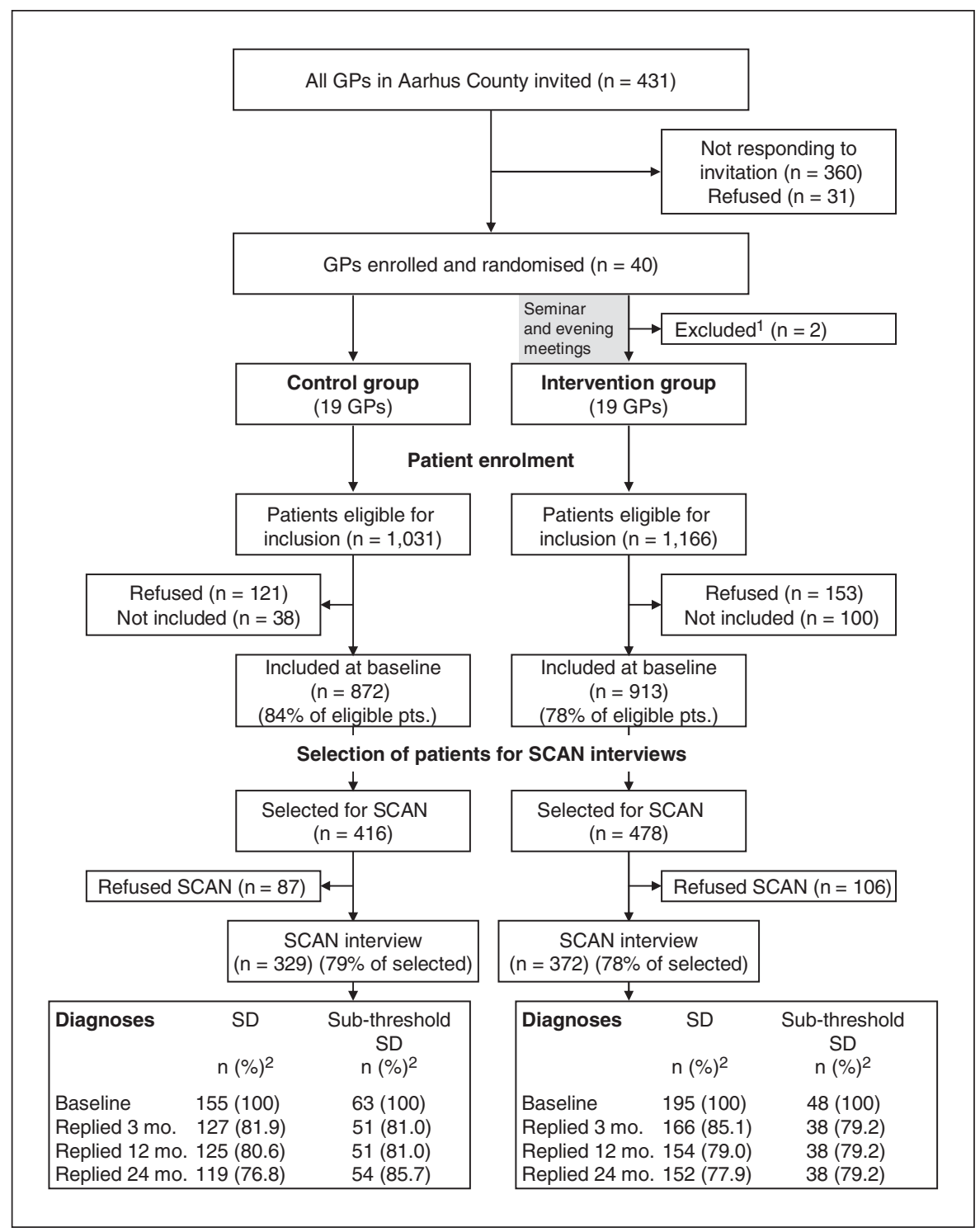

on the screening questionnaire and all previous answers to items for the subscale scores and all previous component scores. Inferences from the imputed datasets were combined to obtain one estimate and likewise one standard error for the parameters of interest [36].

We used Stata [37] and SPSS [38] for the statistics and Solas [36] for generating multiple imputed data sets.

\section{Study Approval}

This study was approved by the Science Ethics Committee in Aarhus County, the Data Surveillance Authority and the Scientific Research Evaluation Committee of the Danish College of General Practitioners.

\section{Results}

\section{Baseline Characteristics and Flow}

No differences were found between the 38 participating GPs and the 393 non-participating GPs with regard to age, gender, type of clinic or postgraduate psychiatric training, but more of the participating GPs had completed courses in doctor-patient communication (52.8 vs. 39.7\%; LR test: $\mathrm{p}=0.045$ ) and they had been working in primary care for fewer years than non-participating GPs (mean: 10.3 vs. 14.1 years; LR test: $\mathrm{p}=0.005$ ). We found no differences between the intervention and control groups.
Toft/Rosendal/Ørnbøl/Olesen/ Frostholm/Fink 


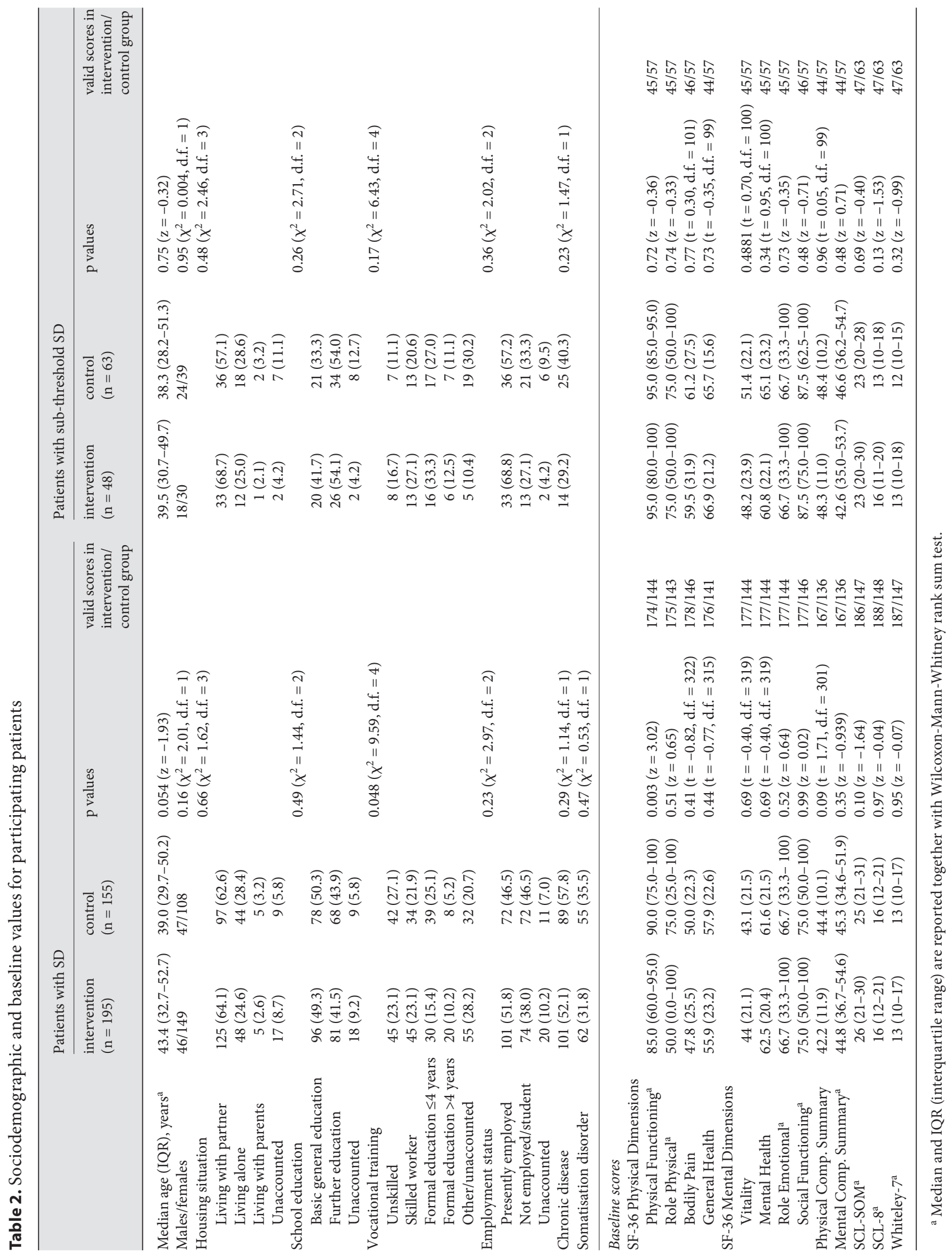




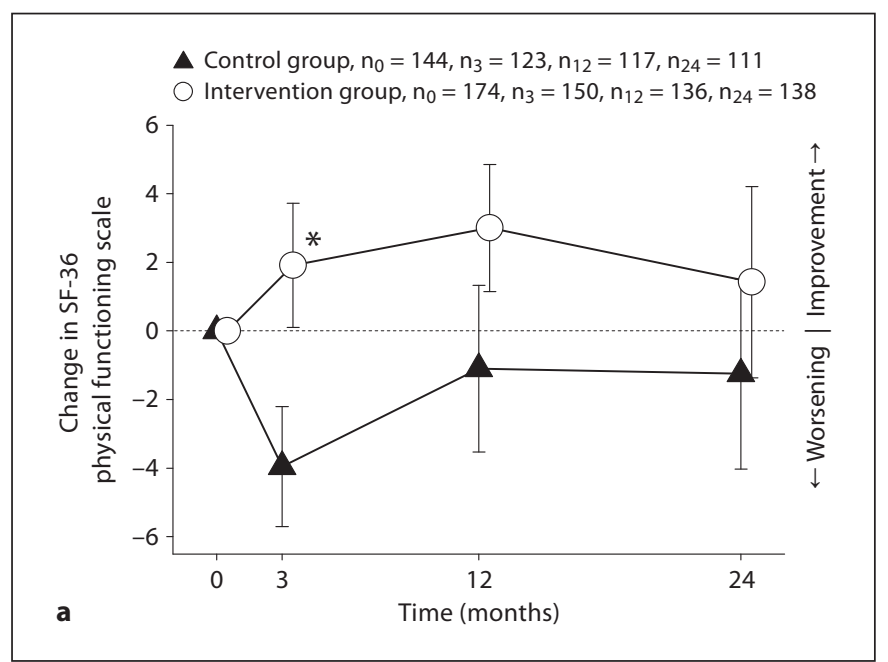

Fig. 2. Descriptives of change in physical functioning during the 2-year follow-up (SF-36 physical functioning scale). Results are means and 95\% CI. a Patients with SD $(n=235-259) .{ }^{*} \mathrm{p}=0.004$. b Patients with somatisation disorder $(n=77-82)$. c Patients with sub-threshold SD ( $n=86-93)$. Significance levels reflect the results based on the linear regression of change in physical functioning on intervention adjusted for patient age, gender, chronic disease and baseline score and corrected for the cluster effect at the GP level.

Of the 2,197 consecutive patients eligible for inclusion, 412 refused or did not participate for other reasons (fig. 1). A total of 1,785 patients $(81.2 \%)$ were included in the Functional Illness in Primary Care study. The mean age of the included patients was 38.8 years (standard deviation 12.9), compared to 42.2 years (standard deviation 13.1) for those who declined participation $(\mathrm{p}<0.001$, Wilcoxon-Mann-Whitney rank sum test), whereas there was no gender difference. The patients not included for other reasons did not differ from the included patients with regard to age or gender.

With regard to participation in the SCAN interview, men refused more often than women ( 27.0 vs. $18.5 \%$; LR test: $p=0.002$, controlled for age), and younger more often than older patients ( 37.5 vs. 40.3 years; LR test: $p=$ 0.005 , controlled for gender). The age and gender of refus-
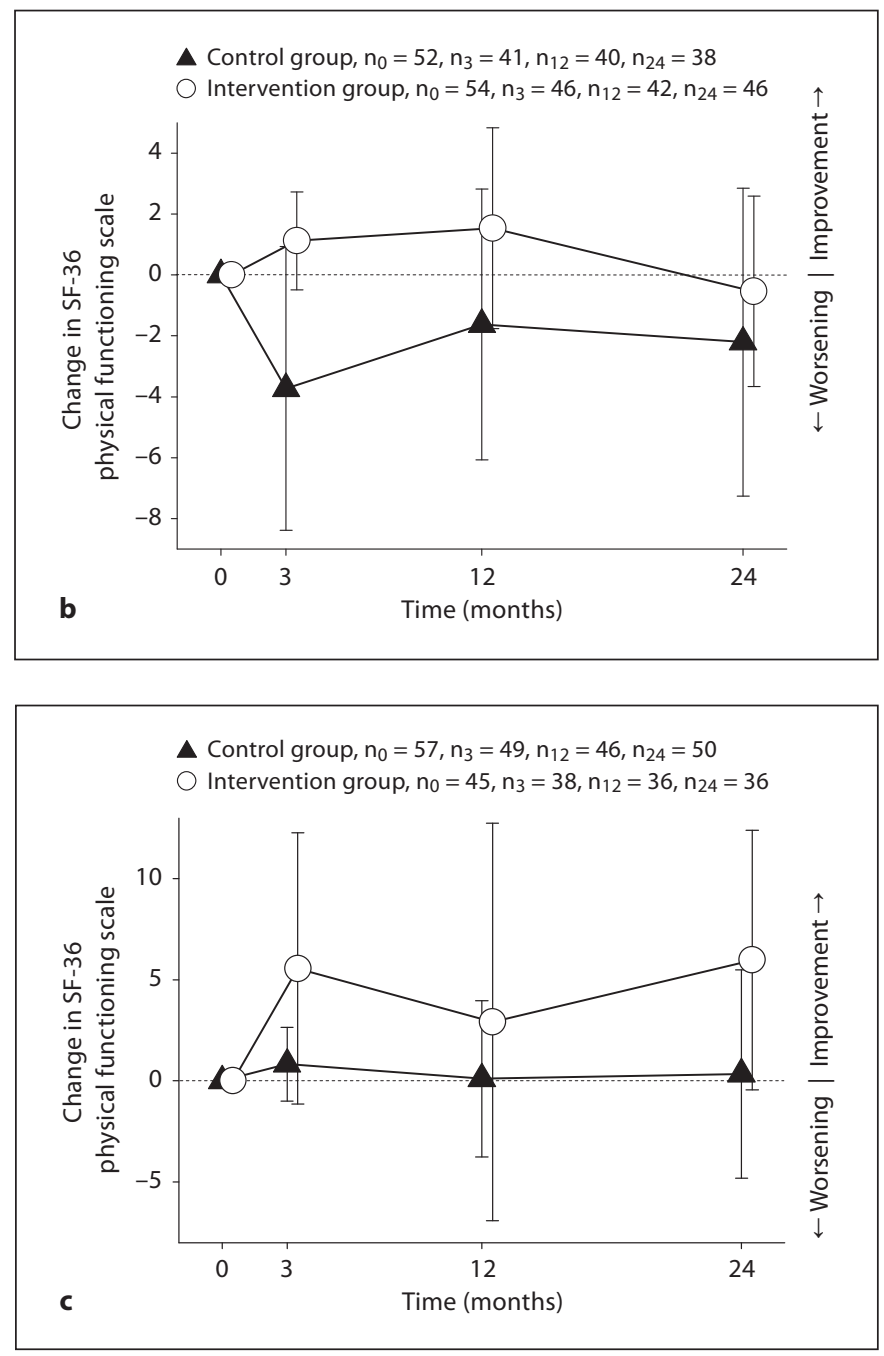

ers did not differ between the intervention and control groups. Based on the SCAN interview, 350 patients were diagnosed as having an ICD-10 SD, of whom 117 had somatisation disorder (F45.0). An additional 111 patients had sub-threshold SD. These were the groups included in this study.

Baseline values were comparable for intervention and control patients, except for the patients with SD in the intervention group, who were more skilled and had lower scores on the SF-36 physical functioning subscale (median 85.0 vs. 90.0 for the control group; $\mathrm{p}=0.003$ ) (table 2). The proportion and characteristics of non-respondents during follow-up did not differ between the intervention and control groups. 


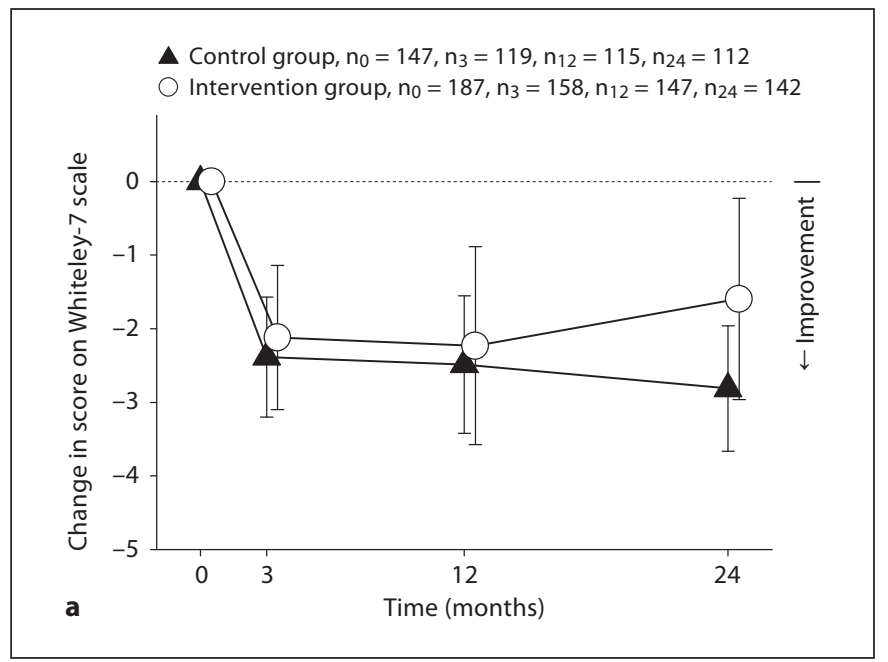

Fig. 3. Descriptives of change in health anxiety during the 2-year follow-up (Whiteley-7). Results are means and 95\% CI. a Patients with SD $(n=289)$. b Patients with somatisation disorder $(n=91)$. ${ }^{*} p<0.001$. c Patients with sub-threshold SD $(n=93)$. Significance levels reflect the results based on the linear regression of change in health anxiety on intervention adjusted for patient age, gender, chronic disease and baseline score and corrected for the cluster effect at the GP level.

\section{Patient Outcome}

With regard to our primary outcome, patients with SD (fig. 2a) had a greater improvement in physical functioning in the intervention group compared with controls, but this was only statistically significant at the 3 -month follow-up (mean difference $4.3,95 \%$ confidence interval [CI] $1.5-7.1 ; \mathrm{p}=0.004)$. The difference in improvement between the intervention and control groups did not reach statistical significance at any point for those patients with somatisation disorder or sub-threshold SD (fig. 2b, c). On the remaining dimensions of the SF-36, there were no differences between groups for patients with SD or subthreshold SD. Patients with somatisation disorder did not differ between groups either, except for vitality at 24 months, where the improvement was smaller in the intervention group than in the control group (mean difference
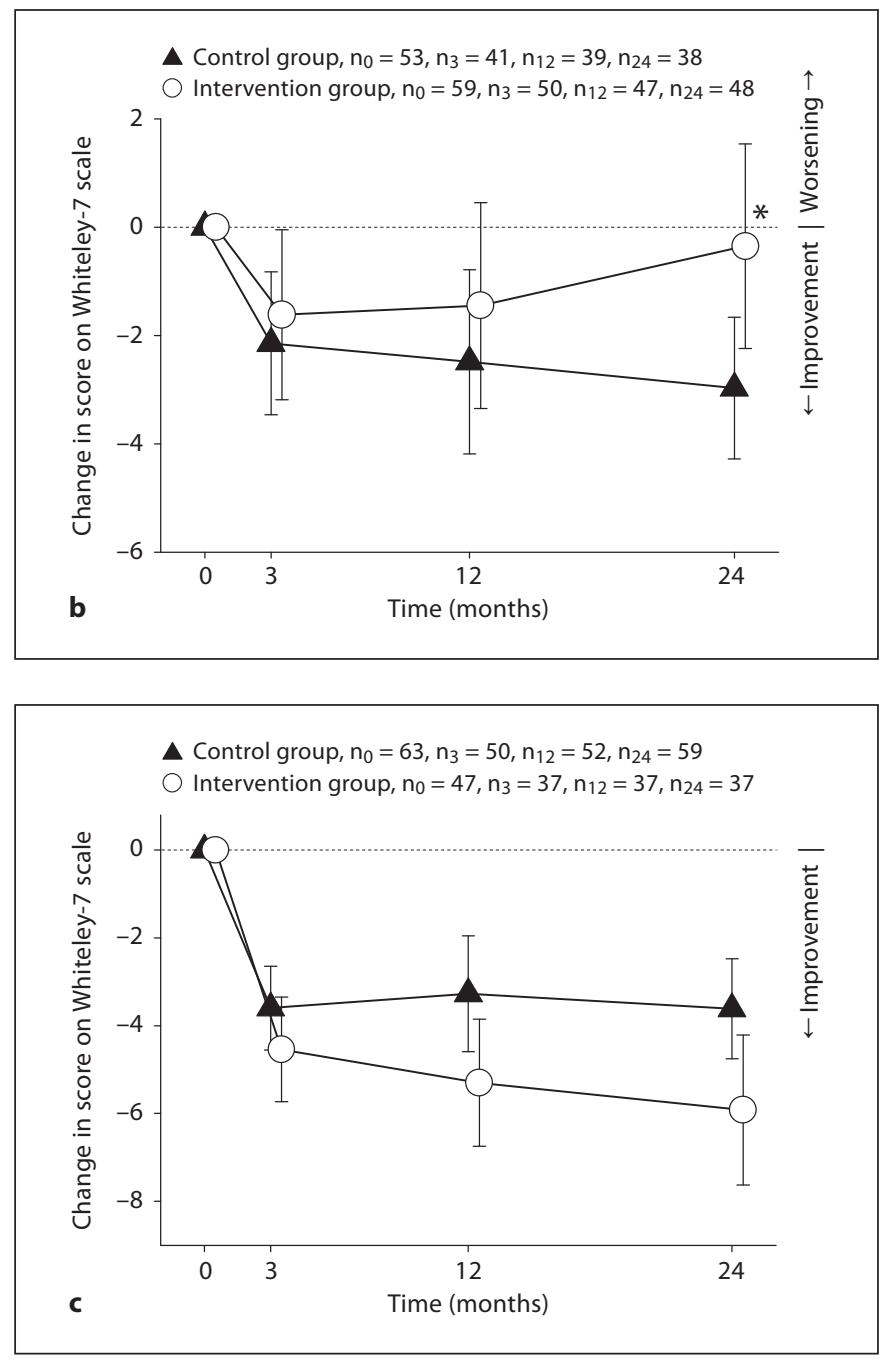

$-10.7,95 \%$ CI -18.8 to $-2.6 ; \mathrm{p}=0.011)$. No differences were found between the groups on the SF-36 component summaries for any of the diagnostic groups.

The subgroup of patients with somatisation disorder in the intervention group had a statistically significantly smaller improvement in health anxiety as measured by the change in the Whiteley-7 score at 24 months compared with the control group (mean difference 3.0, 95\% CI 0.8-3.9; p < 0.001) (fig. 3b). In contrast, patients with sub-threshold SD in the intervention group had a greater improvement in health anxiety than the controls at follow-up, but this difference only reached statistical significance at a $10 \%$ level and only at 24 months (mean difference $-1.1,95 \% \mathrm{CI}-2.3$ to $0.2 ; \mathrm{p}=0.096$ ) (fig. 3c).

Patients in the intervention group with SD and somatisation disorder experienced a smaller improvement in 
the number of physical symptoms than patients in the control group at 24 months (SD group: mean difference 2.0,95\% CI 0.1-3.9, $\mathrm{p}=0.04$; somatisation disorder group: mean difference $3.4,95 \%$ CI $0.1-6.6, p=0.043$ ).

We observed no statistically significant differences in outcome between the groups with regard to anxiety and depression as measured by the SCL- 8 scale.

With regard to the results reported above, only patients who had answered all the items in the questionnaire (respondents) were included in the analyses (approach 1). When we applied the 2 other approaches for handling missing data (cf. paragraph 5, Statistical Methods), slightly smaller differences in changes emerged for all outcomes. With regard to physical functioning in patients with SD at 3 months, the difference decreased from a mean difference of 4.3 (95\% CI 1.5-7.1; $\mathrm{p}=0.004)$ to 3.9 (95\% CI $1.5-6.2 ; \mathrm{p}=0.002)$ and 2.5 (95\% CI -0.4 to 5.4 ; $\mathrm{p}=0.093)$ for the 2 approaches, respectively. For the remaining outcomes and diagnostic groups, the same picture emerged, and the differences in changes in vitality and health anxiety remained statistically significant in patients with somatisation disorder at 24 months using both approaches for handling missing data.

\section{Discussion}

\section{Principal Findings}

Training GPs in the treatment of FSS may have a positive effect on physical functioning in patients with SDs, although we only detected minor changes and a statistically significant effect at the 3-month follow-up. Neither in the subgroup of patients with somatisation disorder nor in the patients with sub-threshold SD did we find a significantly better outcome for physical functioning in the intervention group than in the control group. Patients with somatisation disorder seemed to experience some 'negative effects' on secondary outcome measures, since the improvement was smaller for vitality, health anxiety and the number of physical symptoms during follow-up in the intervention group compared with the control group.

\section{Strengths and Weaknesses of the Study}

The present study was a large, rigorously designed RCT with randomisation at the practice level. The patients were diagnosed by the state-of-the-art interview SCAN, performed by trained psychiatric doctors. The study included consecutive patients who were followed up during a 2-year period with standardised questionnaires.
The training programme in this study aimed to provide GPs without prior training in psychological treatment with skills in the treatment of patients with FSS within the limits of routine practice. The programme has been described in detail elsewhere [25] to guarantee transparency and reproducibility. Previous studies have demonstrated the feasibility of the model and a positive change in GPs' attitudes towards somatising patients [26], as well as patient satisfaction with treatment [39]. A weakness of this study is that we do not know to which extent the GPs actually applied the skills and techniques they were taught and in which individuals they intervened. We analysed the subgroup of patients recognised by GPs as cases at baseline. These cases generally achieved larger numerical outcomes than non-recognised cases, but the results generally fell short of statistical significance due to the weakness of the statistical test power.

Two conditions may have weakened the effect of the intervention. First, although we found the participating GPs to be representative of the county GPs with regard to most variables, we cannot discount the possibility that participating GPs may have had a special interest in FSS. This could have caused a ceiling effect. Second, the extensive assessment of the patients by means of questionnaires and the SCAN inevitably draws attention to psychosocial factors in the patients' illnesses. This may in itself have a curative effect as it becomes evident to the patient that the problem may primarily be of non-organic nature [40].

For the primary outcome variable physical functioning, the baseline value differed between the intervention and control groups. This difference was not present among the non-somatoform patients, which implies that the inclusion rate of severe somatoform cases was probably higher in the intervention group than in the control group. Secretaries of GPs in the intervention group may have included more and severer somatoform patients during the 3-week inclusion period, either using more persuasiveness upon inclusion or, contrary to specific instructions, intentionally scheduling more somatoform patients during the inclusion period. Furthermore, they may have been inclined to allot patients presenting with FSS to the trained GPs in partnership practices where only some of the GPs participated in the study. This possible selection bias may have imposed a disadvantage on the intervention group, as our data show smaller numerical changes in score over time on most variables for the more disabled patients in both study groups.
Toft/Rosendal/Ørnbøl/Olesen/ Frostholm/Fink 
Taking missing data into account, the analysis did not point to any potential skewing of results between the intervention and control groups during follow-up but revealed slightly smaller differences between groups.

We included 13 secondary outcome measures, which imposes a risk of type 1 errors on the secondary outcome measures.

\section{Comparison with Other Studies}

We found 6 comparable studies using GPs as therapists for patients with FSS. Morriss et al. $[18,24]$ trained GPs in the original reattribution model for $8 \mathrm{~h}$. They demonstrated reduced health care costs and improved global (physical) function in patients with depression or anxiety disorders presenting with somatic symptoms. However, the study was a prospective before-and-after study and did not include somatising patients without depression or anxiety disorders. In a more recent RCT by Morriss et al. [19], they trained GPs in reattribution during a 6-hour course. They included patients with physical symptoms of 3 months' duration or longer without medical explanation according to the GPs. Diagnoses were not established by diagnostic interviews. The study did not show any improvement in patient outcome during the 3-month follow-up. In the Netherlands, Blankenstein [20] conducted a small RCT with 20-hour GP training in a modified reattribution model. The results showed that patients had better subjective health as measured on 7 visual analogue scales, reduced health care utilisation and less self-reported sick leave. The study randomly admitted a maximum of 10 frequent attenders with FSS per practice to be treated by the GPs. The patients were not diagnostically interviewed, and analysis of outcome according to different severities of $\mathrm{SD}$ was not possible. Contrary to the study by Blankenstein [20], we measured results independently of GP recognition. This may weaken the results of our study compared with the study of Blankenstein [20], but at the same time it makes our study more pragmatic and more readily transferable to routine clinical practice. In a parallel Danish RCT, 38 GPs were included and trained in the TERM model, and 911 patients with a high score on the SCL-SOM or the Whiteley-7 index for health anxiety were included in the study [22]. At 1-year follow-up, there was no significant improvement in patient outcomes. In contrast to that study, we only included patients with an established diagnosis of either SD or subthreshold SD. In a German RCT, patients were likewise identified by high scores on a symptom questionnaire or the General Health Questionnaire-12 [21]. The GPs were trained according to a modified reattribution model. In contrast to our study, patients showed an improvement in physical symptoms but no improvement in physical health as measured by the SF-12. Finally, a Spanish RCT [23] including 39 GPs and 156 patients indicated a better effect of a GP training programme focusing on communication skills and giving the patients a physical explanation compared to GP training in the traditional reattribution model. However, the duration of the reattribution training was only $3 \mathrm{~h}$, compared to $20 \mathrm{~h}$ for the alternative training. The GPs selected the patients retrospectively, including patients with 4-6 or more medically unexplained lifetime symptoms, which is in contrast to the present study in which consecutive prospective patients were included. We also included biological model explanations in our TERM training, and these models represent a much broader approach, not only focusing on hormone disturbances as in the Spanish study. In the Spanish study, diagnoses only relied on the GPs' assessment.

The TERM programme has been readily accepted by Danish GPs, and the course is now included as part of the compulsory specialist training for GPs in one third of Denmark. This indicates that the GPs find the training useful and that it fills in gaps in the GPs' knowledge and skills as to the management of FSS. However, we could not show a marked improvement in patient outcome in the present study. This discrepancy between clinical popularity and improved process outcome on the one side, opposed to no or minor effects on patient outcome on the other, may raise the question of whether the applied methodology is too crude (i.e. inclusion of all consecutive patients) or whether the applied outcome measures are inappropriate for this kind of study.

\section{Unanswered Questions and Future Research}

Pragmatic studies are needed to evaluate the costeffectiveness and cost-benefit of GP training to secure optimal treatment and management of patients with FSS in primary care. To fulfill this, we need to (1) evaluate and improve the most efficient elements in current intervention models for primary care, (2) develop a stepwise care approach with cooperation between health care sectors $[41,42]$, (3) improve classification of functional symptoms, especially milder cases in primary care [41, 43-45], and finally (4) develop more sensitive patient outcome measures for FSS. 


\section{Acknowledgments}

We are grateful to all the participating GPs and their clinical staff for their effort to inform patients and provide information throughout the study. We thank the participating interviewers (Tønnes Hilden, Karin Clemmensen, Ellen Zeuthen Jeppesen, Lene Høegh, and Birthe T. Hansen). Finally, we also thank the
Interdisciplinary Research Programme of the Danish National Research Council (Sundhedsfremme og forebyggelsesforskning; grant number 9801278) and the Health Service of Aarhus County (project number 0871) for their financial support of the study. The funding sources had no role in the study and did not influence the data collection, data analyses or publications.

\section{References}

${ }_{1}$ Kroenke K, Mangelsdorff AD: Common symptoms in ambulatory care: incidence, evaluation, therapy, and outcome. Am J Med 1989;86:262-266

2 Üstün TB, Sartorius N: Mental Illness in General Health Care: An International Study. Chichester, Wiley, 1995.

- 3 Toft T, Fink P, Oernboel E, Christensen K, Frostholm L, Olesen F: Mental disorders in primary care: prevalence and co-morbidity among disorders. Results from the functional illness in primary care (FIP) study. Psycho Med 2005;35:1175-1184.

-4 Fink P, Sorensen L, Engberg M, Holm M, Munk-Jorgensen P: Somatization in primary care. Prevalence, health care utilization, and general practitioner recognition. Psychosomatics 1999;40:330-338.

5 Smith GR Jr, Monson RA, Ray DC: Patients with multiple unexplained symptoms. Their characteristics, functional health, and health care utilization. Arch Intern Med 1986;146: 69-72.

-6 Buffington CA: Developmental influences on medically unexplained symptoms. Psychother Psychosom 2009;78:139-144.

-7 Ferrari S, Galeazzi GM, Mackinnon A, Rigatelli M: Frequent attenders in primary care: impact of medical, psychiatric and psychosomatic diagnoses. Psychother Psychosom 2008;77:306-314.

$\checkmark 8$ Katon W, Lin E, Von Korff M, Russo J, Lipscomb P, Bush T: Somatization: a spectrum of severity. Am J Psychiatry 1991;148:3440.

-9 Fink P: Surgery and medical treatment in persistent somatizing patients. J Psychosom Res 1992;36:439-447.

10 Porcelli P, Bellomo A, Quartesan R, Altamura M, Iuso S, Ciannameo I, Piselli M, Elisei S: Psychosocial functioning in consultationliaison psychiatry patients: influence of psychosomatic syndromes, psychopathology and somatization. Psychother Psychosom 2009;78:352-358.

11 Bass C, May S: Chronic multiple functional somatic symptoms. BMJ 2002;325:323-326.

12 Kerwick S, Jones R, Mann A, Goldberg D: Mental health care training priorities in general practice. Br J Gen Pract 1997;47:225227.
13 Kroenke K, Swindle R: Cognitive-behavioral therapy for somatization and symptom syndromes: a critical review of controlled clinical trials. Psychother Psychosom 2000;69: 205-215.

14 Schweickhardt A, Larisch A, Wirsching M, Fritzsche K: Short-term psychotherapeutic interventions for somatizing patients in the general hospital: a randomized controlled study. Psychother Psychosom 2007;76:339346.

15 Stuart S, Noyes R Jr: Interpersonal psychotherapy for somatizing patients. Psychother Psychosom 2006;75:209-219.

16 van der Feltz-Cornelis CM, van Oppen P, Ader HJ, van Dyck R: Randomised controlled trial of a collaborative care model with psychiatric consultation for persistent medically unexplained symptoms in general practice. Psychother Psychosom 2006;75: 282-289.

17 Raine R, Haines A, Sensky T, Hutchings A, Larkin K, Black N: Systematic review of mental health interventions for patients with common somatic symptoms: can research evidence from secondary care be extrapolated to primary care? BMJ 2002;325:1082.

18 Morriss R, Gask L, Ronalds C, DownesGrainger E, Thompson H, Leese B, Goldberg $D$ : Cost-effectiveness of a new treatment for somatized mental disorder taught to GPs. Fam Pract 1998;15:119-125.

19 Morriss R, Dowrick C, Salmon P, Peters S, Dunn G, Rogers A, Lewis B, Charles-Jones H, Hogg J, Clifford R, Rigby C, Gask L: Cluster randomised controlled trial of training practices in reattribution for medically unexplained symptoms. Br J Psychiatry 2007; 191:536-542.

20 Blankenstein AH: Somatising Patients in General Practice. Reattribution, a Promising Approach; PhD dissertation, Vrije Universiteit, Amsterdam, 2001.

-21 Larisch A, Schweickhardt A, Wirsching M, Fritzsche K: Psychosocial interventions for somatizing patients by the general practitioner: a randomized controlled trial. J Psychosom Res 2004;57:507-514
22 Rosendal M, Olesen F, Fink P, Toft T, Sokolowski I, Bro F: A randomized controlled trial of brief training in the assessment and treatment of somatization in primary care: effects on patient outcome. Gen Hosp Psychiatry 2007;29:364-373.

23 Aiarzaguena JM, Grandes G, Gaminde I, Salazar A, Sanchez A, Arino J: A randomized controlled clinical trial of a psychosocial and communication intervention carried out by GPs for patients with medically unexplained symptoms. Psychol Med 2007;37:283-294.

24 Morriss RK, Gask L, Ronalds C, DownesGrainger E, Thompson H, Goldberg D: Clinical and patient satisfaction outcomes of a new treatment for somatized mental disorder taught to general practitioners. Br J Gen Pract 1999;49:263-267.

25 Fink P, Rosendal M, Toft T: Assessment and treatment of functional disorders in general practice: the extended reattribution and management model - an advanced educational program for nonpsychiatric doctors. Psychosomatics 2002;43:93-131.

26 Rosendal M, Bro F, Fink P, Christensen KS, Olesen F: Diagnosis of somatisation: effect of an educational intervention in a cluster randomised controlled trial. Br J Gen Pract 2003;53:917-922.

27 Rosendal M, Bro F, Sokolowski I, Fink P, Toft $\mathrm{T}$, Olesen F: A randomised controlled trial of brief training in assessment and treatment of somatisation: effects on GPs' attitudes. Fam Pract 2005;22:419-427.

28 Frostholm L, Fink P, Oernboel E, Christensen KS, Toft T, Olesen F, Weinman J: The uncertain consultation and patient satisfaction: the impact of patients' illness perceptions and a randomized controlled trial on the training of physicians' communication skills. Psychosom Med 2005;67:897-905.

29 Ware JE Jr, Sherbourne CD: The MOS 36item short-form health survey (SF-36). I. Conceptual framework and item selection. Med Care 1992;30:473-483.

30 Fink P, Jensen J, Borgquist L, Brevik JI, Dalgard OS, Sandager I, et al: Psychiatric morbidity in primary public health care: a Nordic multicenter investigation. I. Method and prevalence of psychiatric morbidity. Acta Psychiatr Scand 1995;92:409-418.
Toft/Rosendal/Ørnbøl/Olesen/ Frostholm/Fink 
$>31$ Derogatis LR, Cleary PA: Confirmation of the dimensional structure of the SCL-90: a study in construct validation. J Clin Psychol 1977;33:981-989.

>32 Fink P, Ewald H, Jensen J, Sørensen L, Engberg M, Holm M, Munk-Jørgensen P: Screening for somatization and hypochondriasis in primary care and neurological in-patients: a seven-item scale for hypochondriasis and somatization. J Psychosom Res 1999;46:261273.

33 Ewing J: Detecting alcoholism: the CAGE questionnaire. JAMA 1984;252:1905-1907.

34 Nielsen JM, Vedsted P, Olesen F: The postgraduate psychiatric training of general practitioners: a questionnaire survey in Aarhus County. Ugeskr Laeger 2002;164:895899.
35 WHO: SCAN. Schedules for Clinical Assessment in Neuropsychiatry, version 2.1. Geneva, World Health Organization, Division of Mental Health, 1998.

36 Solas 3.0 for missing data analysis (computer program), version 3.0. Boston, Statistical Solutions Ltd, 2001.

37 Stata (computer program), version 8.0. College Station, Stata Corp, 2003.

38 SPSS 10.0 for Windows (computer program), version 10.0.7. Cary, SPSS Inc, 2000.

39 Christensen KS, Toft T, Frostholm L, Ørnbøl E, Fink P, Olesen F: The FIP study: a randomised, controlled trial of screening and recognition of psychiatric disorders. $\mathrm{Br}$ J Gen Pract 2003;53:758-763.

40 Christensen KS, Rosendal M, Nielsen JM, Kallerup HE, Olesen F: Educational outreach visits. Choice of strategy for interviewing general practitioners. Ugeskr Laeger 2003;165:1456-1460.
41 Fink P, Rosendal M: Recent developments in the understanding and management of functional somatic symptoms in primary care. Curr Opin Psychiatry 2008;21:182-188.

42 Henningsen P, Zipfel S, Herzog W: Management of functional somatic syndromes. Lancet 2007;369:946-955.

43 Fink P, Rosendal M, Olesen F: Classification of somatization and functional somatic symptoms in primary care. Aust NZ J Psychiatry 2005;39:772-781.

44 Rosendal M, Fink P, Falkoe E, Hansen HS, Olesen F: Improving the classification of medically unexplained symptoms in primary care. Eur J Psychiatry 2007;21:25-36.

45 Wise TN: Diagnostic criteria for psychosomatic research are necessary for DSM V. Psychother Psychosom 2009;78:330-332. 